\title{
Practical tests with MMT and ROUTE/DASH on the transport layer of ATSC 3.0
}

\author{
Allan Seiti Sassaqui Chaubet, \\ George Henrique Maranhão Garcia de Oliveira, \\ Gustavo de Melo Valeira and \\ Cristiano Akamine
}




\title{
Practical tests with MMT and ROUTE/DASH on the transport layer of ATSC 3.0
}

\author{
Allan Seiti Sassaqui Chaubet, George Henrique Maranhão Garcia de Oliveira, Gustavo de Melo \\ Valeira and Cristiano Akamine
}

\author{
Electrical Engineering and Computing Program \\ Mackenzie Presbyterian University \\ Sao Paulo, Brazil
}

\begin{abstract}
allanseiti.chaubet@mackenzista.com.br, george.oliveira@mackenzie.br, gustavo.valeira@mackenzie.br, cristiano.akamine@mackenzie.br
\end{abstract}

\begin{abstract}
The development of new technologies allowed television systems to evolve over time. For the transport layer, the Movie Pictures Expert Group (MPEG) developed several standards to deliver multimedia content, including the MPEG-2 Transport Stream (TS), which has been widely explored for years. However, it was developed before the spread of the internet, which led to the interest in a new standard that could fulfill the needs from a connected world. One of these standards is the MPEG Multimedia Transport (MMT), which has inherited some features from the MPEG-2 TS, adapting them to be compatible with the Internet Protocol (IP). The broadband systems also needed a new standard compatible with Hypertext Transfer Protocol (HTTP), resulting in the development of the MPEG-Dynamic Adaptive Streaming over HTTP (DASH). To deliver DASH on broadcast channels, it was combined with the Real-time Object delivery over Unidirectional Transport (ROUTE) protocol. The Advanced Television Systems Committee 3.0 (ATSC 3.0) adopted many technologies to attend the requirements for the next generation of television systems, including both MMT and ROUTE/DASH. This paper presents a historic background of these delivery methods, as well as a brief technical review, focusing on a practical setup to test the methods mentioned, analyzing the differences and similarities of their properties.
\end{abstract}

Index Terms-MMT, ROUTE/DASH, ATSC 3.0, Transport protocols.

\section{INTRODUCTION}

$\mathrm{N}$ EW television systems have been developed to be compatible with new technologies, either for better user experience or better broadcasting conditions. From black and white to color systems, from analog to digital, allowing better video and audio quality, error detection and correction methods, power and spectrum efficiency and different antenna configurations.

For the next television generation, some of the expected improvements are the transmission in Ultra High Definition (UHD), immersive audio, internet compatibility, hybrid services, personalized content and interactivity. For that, improvements on the transport layer were needed.
The Moving Pictures Expert Group (MPEG), has developed several standards for multimedia content delivery, such as the MPEG-2 Transport Stream (TS), which was adopted in many broadcast systems, including the Advanced Television Systems Committee 1.0 (ATSC 1.0), the Digital Video Broadcasting (DVB), and the Integrated Services Digital Broadcasting - Terrestrial (ISDB-T/Tb), some of which are still in operation [1]. Even though MPEG-2 TS has several characteristics that made it appropriate for broadcasting, the popularization of the Internet presented complications for this standard, with the interest in new technologies such as streaming services, Internet Protocol Television (IPTV) and hybrid systems. Thus, it was observed the need to carry out new studies that could result in the proposal of new transport protocols [2].

So, in 2009, MPEG hosted workshops to identify the requirements introduced by the new technologies and change of service environments. One of the major observations was that some features from MPEG-2 TS are still convenient for the next generation of television systems and therefore should be maintained or upgraded. However, other features should be simplified and adapted to consider the use of Internet Protocol (IP) as its delivery protocol, also requiring compatibility with Hypertext Markup Language 5 (HTML5). This led to the development of MPEG Multimedia Transport (MMT), which was published in 2014 as part 1 of ISO/IEC 23008 [3], [4]. MMT was adopted by the ATSC 3.0 [5] and the Integrated Services Digital Services for Satellite, 3rd generation (ISDB-S3) [6].

In the same year, MPEG noticed a market demand for a new standard for streaming services, that is, for broadband use. The reason for that was that the Real-time Transport Protocol (RTP) started to face difficulties, because it was not supported by many Content Delivery Networks (CDN), which replaced managed IP networks. Other issues were being blocked by firewalls and not being friendly for largescale usage, since it required an individual session for each client. Hypertext Transfer Protocol (HTTP) solves these situations [7].

This led to a Call for Proposals from MPEG for a streaming 
standard based on HTTP, which resulted as the MPEGDynamic Adaptive Streaming over HTTP (DASH) standard. It was published as ISO/IEC 23009 in 2012 and became very popular, being used on YouTube and Netflix, for example [8].

However, HTTP is not appropriate for broadcast delivery, so a new transport method was elaborated to deliver DASH on broadcast channels, which is called Real-time Object delivery over Unidirectional Transport (ROUTE), a work in progress by the Internet Engineering Task Force (IETF). ROUTE has improved certain features from File Delivery over Unidirectional Transport (FLUTE), such as more flexibility to deliver multiple streams at the same time, making ROUTE/DASH a logical extension from FLUTE/DASH [9].

The ATSC 3.0 meets the requirements for the next generation of television systems by combining modern technologies for all its layers. For the transport layer, it adopted both MMT and ROUTE/DASH, making it possible to compare these standards using the same setup, considering the usage rules for this standard.

For this paper, a complete setup for the ATSC 3.0 allowed the analysis of the standards mentioned above. With this practical approach, it was possible to observe the characteristics of each standard on a transport analyzer, considering the usage rules for the ATSC 3.0.

This paper is organized in six sections. Sections II, III and IV give a brief overview on the ATSC 3.0, MMT and ROUTE/DASH, respectively. Section V explains the setup used and contains the analysis made. Section VI presents the conclusion of this work.

\section{ATSC 3.0}

The ATSC 3.0 has been developed to meet the requirements for the next generation of broadcast systems. It has been designed in a layered architecture, making flexibility and extensibility easier. The three layers that define the system are the Physical layer, the Management and Protocols layer, and the Application and Presentation layer [10].

The physical layer has been built with some the latest technologies available at the moment, such as Low-Density Parity Check (LDPC) codes, modulation with non-uniform constellations (NUCs), Layered Division Multiplexing (LDM), Multiple-Input Single-Output (MISO) and MultipleInput Multiple-Output (MIMO) frame types and channel bonding. The broadcaster can set different configurations that can suit for several scenarios, aiming for more robustness or higher bitrates [8].

The Management and Protocols layer groups two system layers, becoming the transport layer of ATSC 3.0, which is based on IP and is responsible for the delivery of services and content over broadcast, broadband and hybrid networks. Signalling, media synchronization and application-layer forward error correction (AL-FEC) are also included in this layer. The methods specified for service delivery are the MMT and the MPEG-DASH. MMT delivers Media Processing Units (MPUs) using the MMT protocol (MMTP), while MPEG-DASH delivers DASH Segments using the ROUTE protocol. ROUTE is also used to deliver content that does not require real-time rendering. Both MMTP and/or ROUTE can be used for signalling delivery, and the Service List Table (SLT) provides the Bootstrap Signalling information. DASH is also used on the broadband side for the delivery of hybrid services. Figure 1 shows a conceptual model for the system.

\begin{tabular}{|c|c|c|c|c|c|c|}
\hline \multicolumn{7}{|c|}{ Applications (HTML5/JS/native) } \\
\hline MPU & \multirow{2}{*}{ 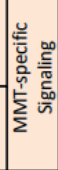 } & \multirow{2}{*}{$\begin{array}{l}\text { NRT } \\
\text { Files }\end{array}$} & \multicolumn{2}{|c|}{ DASH Player/Decoders } & \multirow{2}{*}{$\begin{array}{l}\text { NRT } \\
\text { Files }\end{array}$} & \multirow{2}{*}{$\begin{array}{l}\text { All Sig- } \\
\text { naling } \\
\text { Objects }\end{array}$} \\
\hline \begin{tabular}{|c|} 
EME/CENC \\
MPU (ISO BMFF)
\end{tabular} & & & \multicolumn{2}{|c|}{$\begin{array}{c}\text { EME/CENC } \\
\text { DASH Segment (ISO } \\
\text { BMFF) }\end{array}$} & & \\
\hline & & \multicolumn{2}{|c|}{ HTTP Proxy } & \multirow{3}{*}{\multicolumn{2}{|c|}{ HTTP }} & \\
\hline MMTP & & \multirow{2}{*}{\multicolumn{2}{|c|}{ ROUTE }} & & & \\
\hline & SLT & & & & & \\
\hline \multicolumn{4}{|c|}{ UDP } & \multicolumn{2}{|c|}{ TCP } & \\
\hline \multicolumn{7}{|c|}{ IP } \\
\hline \multicolumn{4}{|c|}{ Broadcast } & \multicolumn{3}{|c|}{ Broadband } \\
\hline
\end{tabular}

Fig. 1. Conceptual protocol stack for ATSC 3.0 (ATSC A/331:2020).

ROUTE and MMPT usage shall follow the rules:

- ROUTE and MMTP sessions shall not be used simultaneously for a broadcast delivery of a Linear Service without an app-based feature.

- At least one ROUTE session shall be used for a broadcast delivery of a Linear Service with an appbased feature (additional ROUTE and MMTP sessions are allowed, except for streaming media components in the same service).

- Only ROUTE sessions shall be used for a broadcast delivery of an app-based service, an Electronic Service Guide (ESG) Service, an Emergency Alert (EA) Service, or a Digital Rights Management (DRM) Data Service [9].

These rules consider the following terms: Linear Service, App-Based Feature and App-Based Service.

A Linear Service is a service that consists of at least one continuous video component associated with at least one continuous audio component which is then associated with at least one closed caption component and may contain appbased features.

An App-Based Feature is an application that can be directed to take individual actions on specific times using optional files and notifications.

An App-Based Service is a service that consists completely of app-based features, providing interface with the service for the user.

The application and presentation layer consists of the technologies for Audio/Video (A/V) coding, interactivity, accessibility, captions, subtitles, and other services [12].

\section{MMT}

As stated before, the development of MMT started because MPEG-2 TS faced complications for personalized content and hybrid systems. However, it has inherited some features from MPEG-2 TS. One of its main characteristics is the ability to multiplex multiple streams in a single stream, interleaving data packets to allow a synchronized playback.

The MMT standard [13] defines the tools to encapsulate, organize, signal and deliver media and data. Its architecture is composed of three functional areas: the MPU format, delivery and signalling, as shown by Figure 2. Depending on the needs of a particular multimedia service, only part of these tools can be used. 

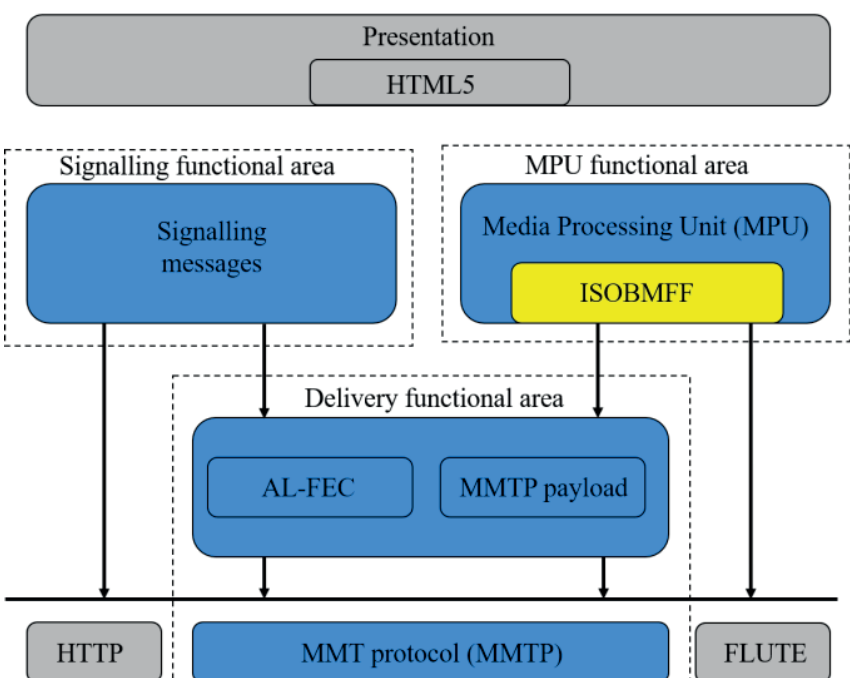

Defined by ISO/IEC 23008-1 $\square$ Out of scope of ISO/IEC 23008-1

Referenced by ISO/IEC 23008-1

Fig. 2. MMT architecture (ISO/IEC 23008-1).

The MPU functional area consists of the logical arrangement of media, Package and the format of data units, using the ISO-based media file format (ISOBMFF). The Package provides the information about the compression on the media content and the format of data units that encapsulates the encoded media. In more detail, a Package is composed of at least one Asset and one Presentation Information (PI).

An Asset contains one or more MPUs with the same Asset identification (ID), that carries timed or non-timed media content, which can be an audio, a video or a web page, for example. Each Asset is associated with an asset delivery characteristics (ADC). A single ADC can be associated with several Assets, but each Asset can only be associated with one ADC. Also, MPUs of timed content contained in the same Asset shall have distinct presentation times.

The PI is a document that specifies how Assets are related for consumption, with freedom for spatial and temporal arrangements, thus providing an improvement from MPEG-2 TS, which assumed a single video and audio synchronized from the beginning in a fixed arrangement. An example of a PI document is the combination of HTML5 and composition information (CI) documents. Another possibility is to use a media presentation description (MPD), which is a manifest that describes the content and its characteristics [4], [7].

Figure 3 presents an overview for the MMT Package.

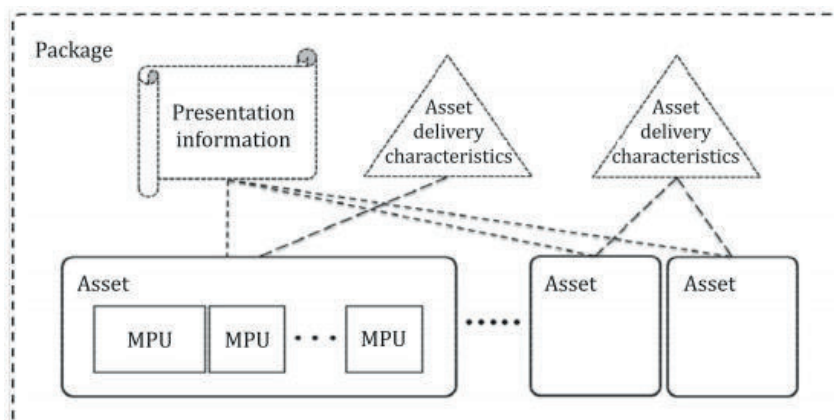

Fig. 3. MMT Package overview (ISO/IEC 23008-1).
The delivery functional area contains the MMTP as the application layer transport protocol and the MMTP payload, which is the payload format and is agnostic to media codecs, therefore supporting new codecs by requiring signalling messages that identify them.

The signalling functional area manages the consumption of media data from the Package and the delivery for the MMTP and the MMTP payload.

\section{ROUTE/DASH}

MPEG-DASH is based on a client-server model to allow adaptive streaming. A HTTP server contains a MPD and the multimedia content that consists of multiple $\mathrm{A} / \mathrm{V}$ segments. The MPD is then delivered to a DASH client, which becomes aware of the content available and its characteristics. After analyzing such information, the DASH client can start streaming any chosen content, being able to change to a different configuration on the next available switching point The system constantly monitors the available bandwidth. Whenever the bandwidth is lower than the current bitrate being streamed, it switches to a lower bitrate at the next available switching point. It can also switch to another bitrate or a different audio content if the user decides to do so.

Figure 4 presents the architecture for MPEG-DASH, which only defines the red blocks. The delivery of the MPD, the encoding format, the heuristics control and the media player are out of the standard's scope [7].

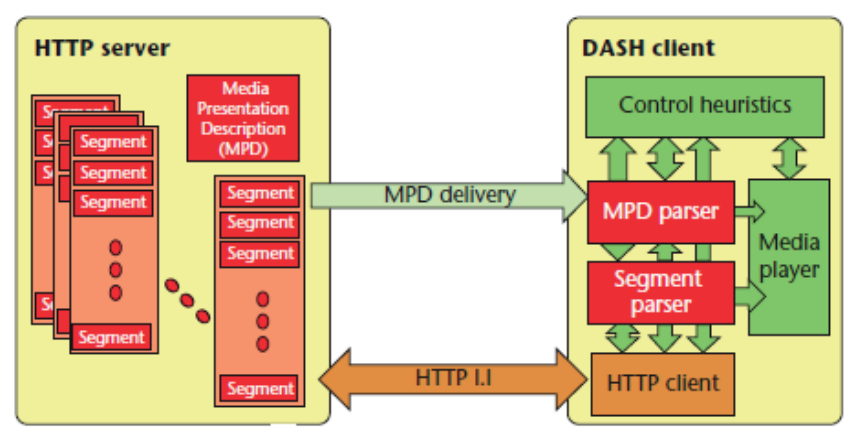

Fig. 4. MPEG-DASH architecture [7].

ROUTE is a delivery method that supports AL-FEC and can be combined with MPEG-DASH to deliver it over broadcast. ROUTE consists of two major components, a source protocol and a repair protocol. The source protocol is used to deliver objects and is aligned with FLUTE but has been optimized for the delivery of real-time media. The repair protocol protects one or multiple objects that are delivered through the source protocol. Source and repair protocols are independent, meaning that the source protocol can be used alone. Using only the repair protocol is also allowed and could be used on specific scenarios where it is expected that all receivers are AL-FEC capable, such as the delivery exclusively for mobile reception and the delivery for targeted regions. Since all packets in ROUTE are Layered Coding Transport (LCT) packets, which are defined in RFC 5651 [14], source and repair packets can be differentiated by the use of distinct ROUTE sessions, distinct LCT channels that use different Transport Session Identifiers (TSIs) values in the LCT header, and the Protocol-Specific Indication (PSI) bits in the LCT when both source and repair protocols are carried in the same LCT channel [9]. 


\section{SETUP AND ANALYSIS}

To test MMT and ROUTE/DASH on the transport layer of ATSC 3.0, the setup presented on Figure 5 was used, where two services are being transmitted at the same time, one with MMTP (identified with Service ID 6001) and the other with ROUTE (identified with Service ID 6002). The description of the equipment is provided below.

The A/V source delivers a content to the encoder, which accepts the following input types: IP, Serial Digital Interface (SDI), SDI 4K, Real Time Messaging Protocol (RTMP), Receiver Demodulator, Zixi, Society of Motion Picture and Television Engineers (SMPTE) 2022-6 and SMPTE-2110. For this setup, a source with the SDI format was used.

The encoder output forwards the $\mathrm{A} / \mathrm{V}$ encoded stream to the multiplexer (MUX) in the Web Distributed Authoring and Versioning (WebDAV) format, which is an extension to HTTP for the execution of content authoring operations remotely [16]. In this stage, signalling information is added and the signal is forwarded to the scheduler (in our setup, the MUX and the scheduler are integrated). The scheduler adds transmitting data in the stream for the modulator, with the intention that the receiver finds the desired services, which is a function of the Service Layer Signalling (SLS) table. The Low Level Signalling (LLS) table is also added, in order to improve the channel scan speed [17].

The scheduler feeds the modulator with Studio-toTransmitter Link (STL) packets, which is an interface between the transport and physical layers [18]. So, the modulator can convert the STL stream in a Radio Frequency (RF) channel, considering the modulation parameters configured.

The transport analyzer can monitor the STL stream from the scheduler or the MMTP and ROUTE output from the MUX, where it is possible to check several information such as the received tables, codec information, bit rate, resolution, frame rate and the audio and video synchronization.

Figure 6 presents part of the signalling configuration of the MUX for the MMT service, showing information about the Assets contained. The Asset type specifies the type of the Asset using a four-character code (4CC), according to the MPEG-4 Registration Authority (MP4RA) [19]. In this setup Asset \#1 was set as High Efficiency Video Coding (HEVC), Asset \#2 was set as Advanced Audio Coding (AAC), and Asset \#3 was set as MPEG-4 Audio (MP4A). The Asset Schema specifies the identification mechanism and either Universally Unique Identifier (UUID) or Uniform Resource Identifier (URI) can be used for MMT. For this setup, all Assets were set as UUIDs. The Default Asset Flag indicates if an Asset should be a default Asset [13].

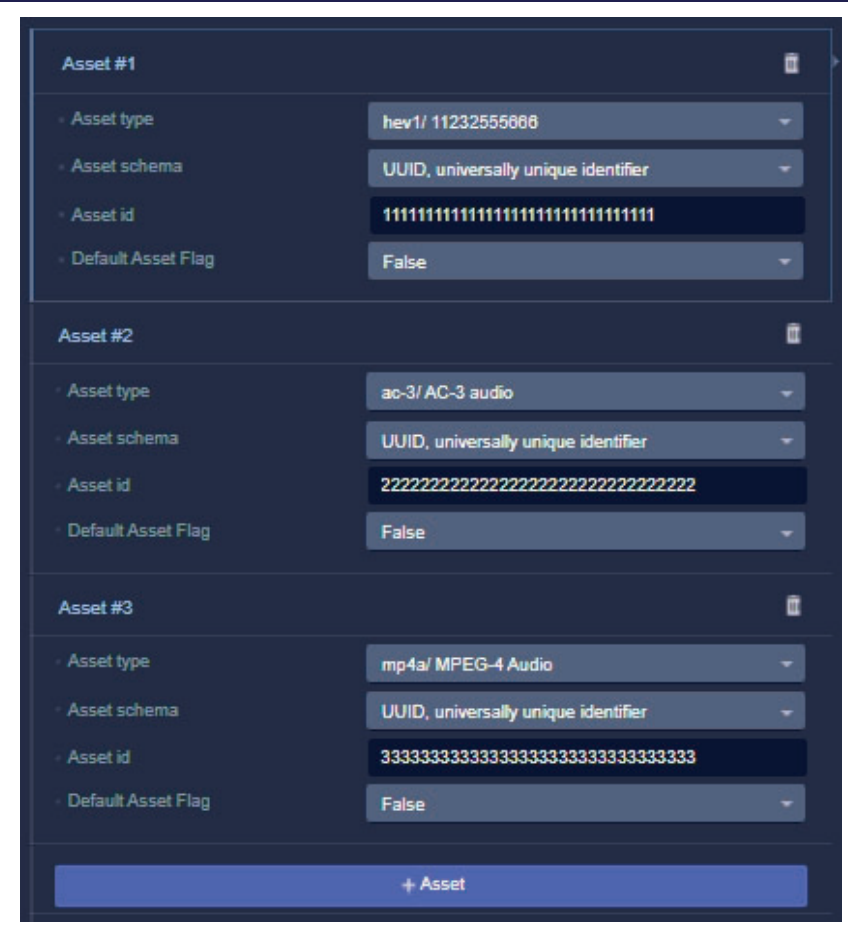

Fig. 6. MUX configuration for the Assets of the MMTP service.

Figures 7 and 8 show the respective configuration for the ROUTE/DASH service, with information of the Route Sessions (RSs) and LCT Sessions (LSs). For the configuration of the Route Sessions, the $s I p A d d r$ sets the IP Address of the source, the $d I p A d d r$ sets the IP Address of the destination, and the dPort sets the destination port. For the LCT Sessions, the TSI must have unique values for each Session. The $B W$ value can set the maximum bandwidth required by the LCT channel [5].

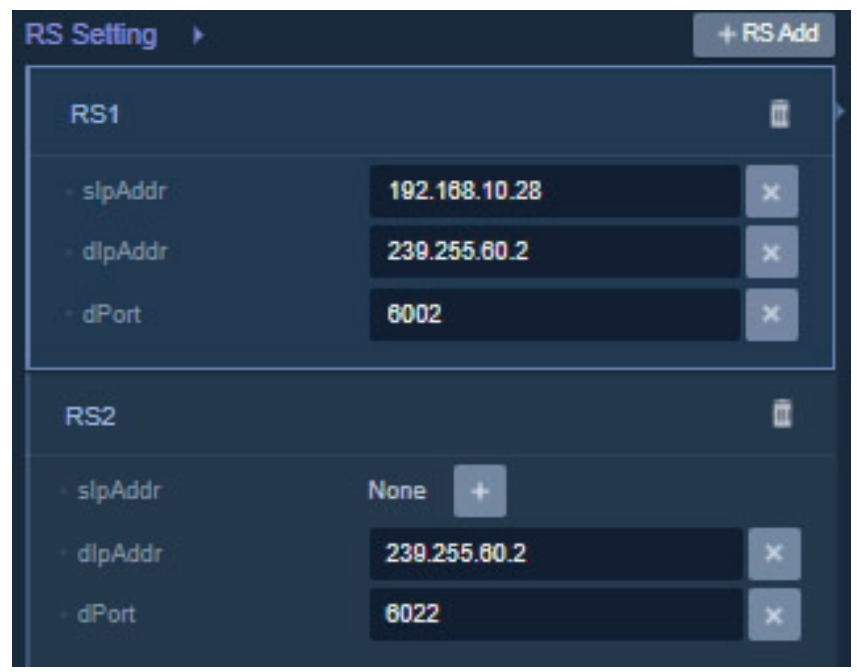

Fig. 7. MUX configuration for the RSs of the ROUTE service.

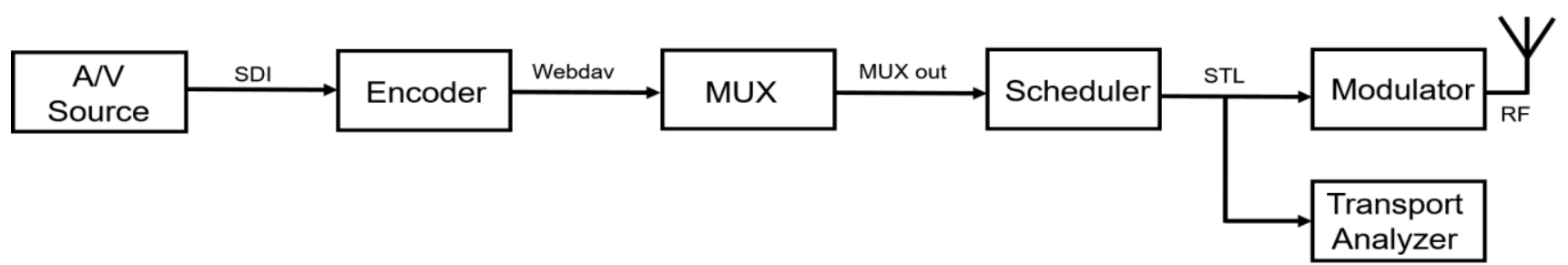

Fig. 5. Setup used to test both MMT and ROUTE/DASH delivery methods. 


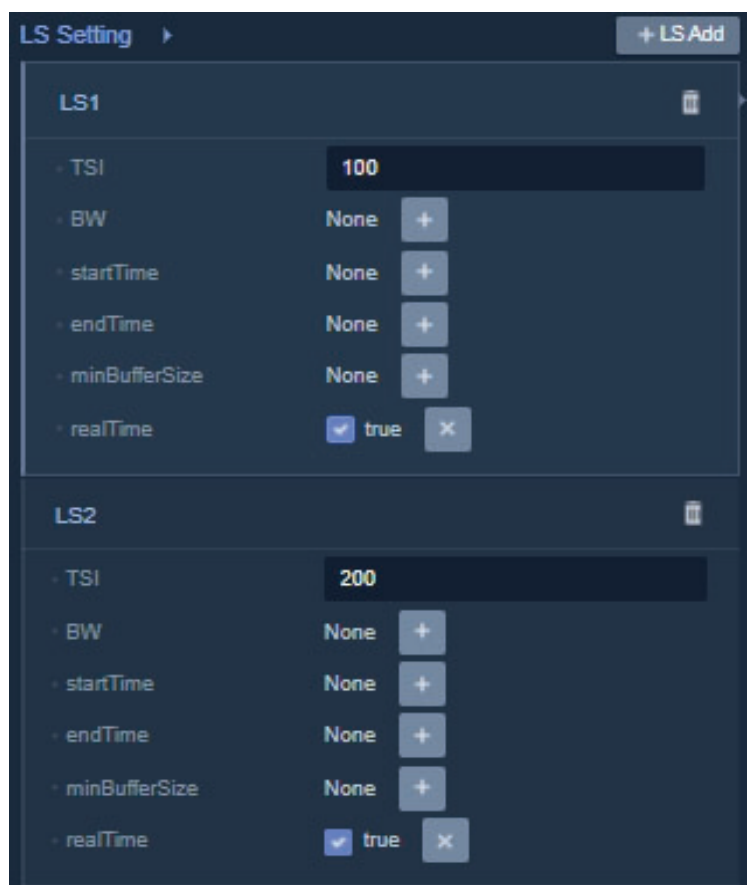

Fig. 8. MUX configuration for the LSs of the ROUTE service.

Figure 9 shows the STL information obtained from the transport analyzer, where the parameters for the Physical layer can be observed. For this setup, the Fast Fourier Transform (FFT) size is $16 \mathrm{~K}$, the Guard Interval (GI) is $7 / 2048$, the modulation order is 64QAM-NUC, FEC is $10 / 15$ and LDM was not used.

\begin{tabular}{|c|c|c|c|}
\hline \multicolumn{4}{|l|}{ STL } \\
\hline & BOOTSTRAP & SUBFRAME & \\
\hline Num_sym_bs & $4 \mathrm{msec}$ & SUBRAME & SUBFRAME 0 \\
\hline Ea_wake_up & 0 & $\begin{array}{r}\text { FFT size } \\
\end{array}$ & $16 \mathrm{~K}$ \\
\hline Min_Time_To_Next & 200 & Reduced carrier & $192^{*}$ red carr in \\
\hline Bandwidth & $6 \mathrm{MHz}$ & Guard interval & G17_2048 \\
\hline Bsr_Coeff & $6 \mathrm{MHz}$ & Numofdm & 90 \\
\hline Preamble_struct & 65 & Spilot pattern & SP3_4 \\
\hline Stl Inner Timing & 2020-12-07T15:14:15 & Spilot boost & 0 \\
\hline \multirow[t]{2}{*}{ Stl Inner Timing } & $75145333 \mathrm{nsec}$ & Sbs first & On \\
\hline & \multirow{2}{*}{$\begin{array}{c}\text { MONITORING } \\
\text { Normal Operation }\end{array}$} & Sbs Last & On \\
\hline State & & Freq interleaver & On \\
\hline DataR & $14.63 \mathrm{Mbps}$ & Sbs num cells & 0 \\
\hline DataT & $18.33 \mathrm{Mbps}$ & Num plp & 1 \\
\hline Alp_pkt_cnt & 5817234 & SUB PLP LIST & SUBFRAME 0 \\
\hline Ip_pkt_cnt & 4685175 & SUB PLP & PLP 0 \\
\hline Lmt_pkt_cnt & 34526 & Id & 0 \\
\hline Stl outer timestamp & $946423 \mathrm{sec}$ & ULS flag & On \\
\hline Stl outer timestamp & $75 \mathrm{msec}$ & Layer & Core layer \\
\hline Mismatch Sequence & 6996 & Start & 0 \\
\hline Matched Preamble & 0 & Size & 1126477 \\
\hline \multicolumn{2}{|l|}{ t } & Fec type & $\mathrm{BCH}+64 \mathrm{~K}$ \\
\hline & PREAMBLE & Mod order & 64QAM-NUC \\
\hline Preamble red carr. & 0 & Code rate & $10 / 15$ \\
\hline & \multirow{2}{*}{232 byte } & Timode & $\mathrm{CTI}$ \\
\hline L1B L1D size & & Ti ext & Off \\
\hline L1B L1D fec type & Mode 1 & Cti depth & 1024 or 1448 \\
\hline L1B time info flag & 3 & Type & non-dispersed \\
\hline L1D time & $2020-12-07 \pi 15: 14: 15075,145.333$ & Num subslice & 1 \\
\hline Frame length mode & symbol-aligned mode & Subslice intvl & 0 \\
\hline Frame length & 0 & Hti cell & Off \\
\hline Num subframe & 0 & Hti inter & Off \\
\hline Xmtr group num & 25 & Hti num ti & 1 \\
\hline & 0 & Hti num fec & 1 \\
\hline Xmtrid & 0 & Hti num fec & 1 \\
\hline Tx time offset & 0 usec & Ldm injection & $0.0 \mathrm{~dB}$ \\
\hline Txid injection level & Off & Data rate & $18.33 \mathrm{Mbps}$ \\
\hline
\end{tabular}

Figure 10 shows the input stream info obtained from the monitor of the transport analyzer, where it can be observed that for this setup, the audio was coded using Advanced AAC at $128 \mathrm{Kbps}$, the video was coded using HEVC at $2.35 \mathrm{Mbps}$. Since the transport analyzer is constantly monitoring the streams, the Total Rate of the streams is different because the images were obtained at different moments, however, the average rate for both streams was around $3.00 \mathrm{Mbps}$. The Signal Rate for each stream also changed during time but not significantly, meaning that the Signal Rate for the ROUTE service was higher.

\begin{tabular}{|c|c|c|c|}
\hline \multirow{3}{*}{$\begin{array}{l}\text { Input Stream Info } \\
\text { ATSC } 3.0 \\
\text { Total Rate }\end{array}$} & \multirow{3}{*}{$\begin{array}{l}\text { MWTP } \\
2.95 \mathrm{Mbps}\end{array}$} & \multirow{3}{*}{$\begin{array}{l}\text { Input Stream Info } \\
\text { ATSC } 3.0 \\
\text { Total Rate }\end{array}$} & \multirow{3}{*}{$\begin{array}{l}\text { ROUTE } \\
\text { 2.71Mbps }\end{array}$} \\
\hline & & & \\
\hline & & & \\
\hline Media Rate & 2.93 Mbps & Media Rate & $2.64 \mathrm{Mbps}$ \\
\hline $\begin{array}{l}\text { Signal Rate } \\
\text { (SLS+LLS) }\end{array}$ & $(17+0) \mathrm{Kbps}$ & $\begin{array}{l}\text { Signal Rate } \\
\text { (SLS+LLS) }\end{array}$ & $(76+0)$ Kbps \\
\hline \multicolumn{2}{|c|}{ Including UDP/IP Header $\mathbf{\square}$} & \multicolumn{2}{|c|}{ Including UDP/IP Header } \\
\hline Codec & AAC & Codec & AAC \\
\hline Bitrate & $128 \mathrm{Kbps}$ & Bitrate & 130 Kbps \\
\hline ChannelNum & 2 & ChanneINum & 2 \\
\hline SamplingRate & $48000 \mathrm{~Hz}$ & SamplingRate & $48000 \mathrm{~Hz}$ \\
\hline $\begin{array}{l}\text { Channel Configuration } \\
\text { (FronUSurr/LFE) }\end{array}$ & 2 & $\begin{array}{l}\text { Channel Configuration } \\
\text { (FrontSurriLFE) }\end{array}$ & 2 \\
\hline Video & & Video & \\
\hline Codec & HEVC & Codec & reve \\
\hline Bitrate & 2.36 Mbps & Bitrate & 2.34 Mbps \\
\hline Resolution & $1024 \times 576$ & Resolution & $1024 \times 576$ \\
\hline Framerate & $59.94 \mathrm{fps}$ & Framerate & $59.94 \mathrm{fps}$ \\
\hline
\end{tabular}

Fig. 10. Input Stream Info from the transport analyzer for both services.

In the setup used in this paper, only the Service List Table (SLT) and System Time signalling are transmitted inside of the LLS, as shown in the Figure 11. The Version is incremented by 1 when a change occurs on the table identified.

\begin{tabular}{l} 
Low Level Signalling (LLS) \\
\begin{tabular}{|c|c|c}
\hline Type & Version & Receiving period \\
\hline SLT & 67 & 500 \\
RRT & & \\
SystemTime & 984 \\
AEAT & 2 & \\
OnscreenMessageNoti... & \\
VIT \\
CPT
\end{tabular} \\
\hline
\end{tabular}

Fig. 11. LLS Signalling information.

The SLT provides the description of the service, identifying the used protocol, major channel (physical channel), minor channel (virtual channel) and SLS destiny information. It shall be repeated in the LLS at least every 5 seconds, however, repeating it more frequently (preferably every second, but not more than that) reduces the channel scan time. Figure 12 presents the content of SLT. It is possible to note that the slsProtocol value is 1 for ROUTE and 2 for MMTP, as determined by the ATSC 3.0 standard [5]. 


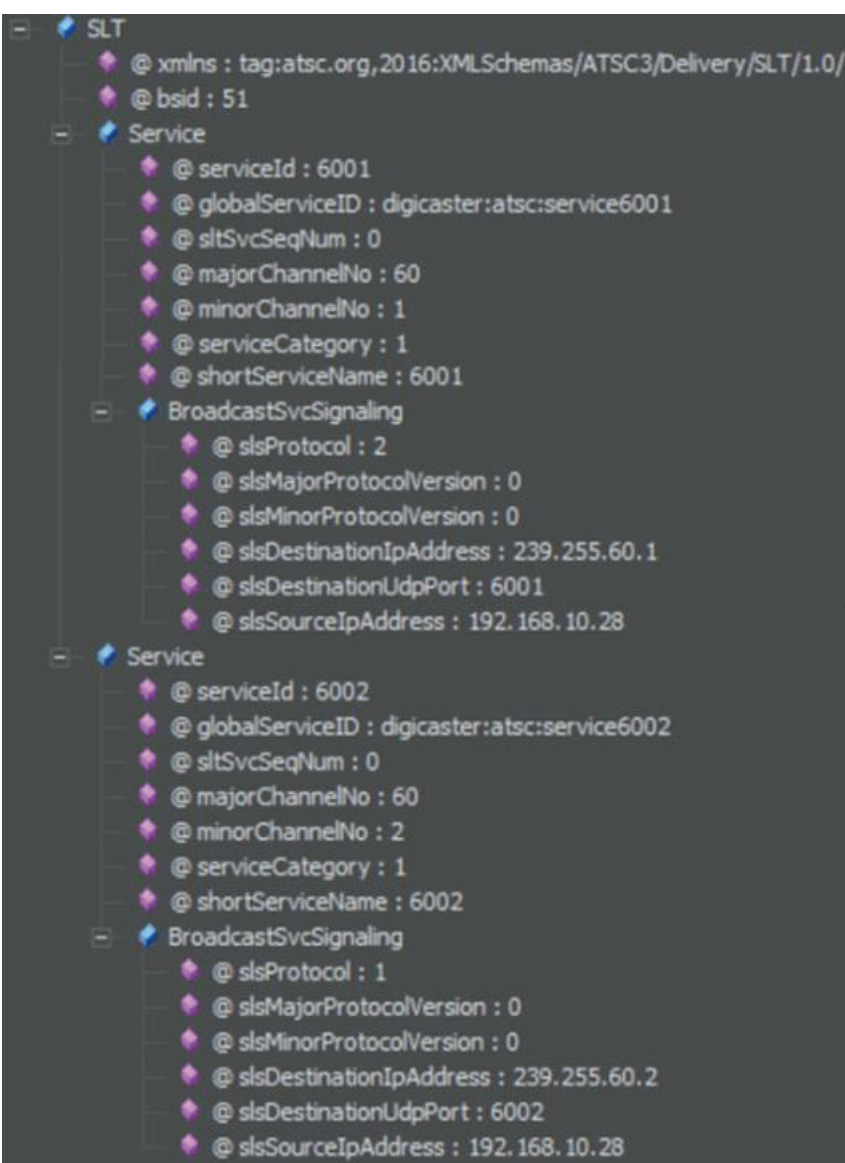

Fig. 12. SLT XML (Extensible Markup Language).

In SystemTime signalling, currentUtcOffset contains the number of leap seconds, utcLocalOffset indicates the offset between Coordinated Universal Time (UTC) and the originating broadcast station's time zone, and dsStatus indicates whether the transmitter location is currently on daylight saving time or not [5].

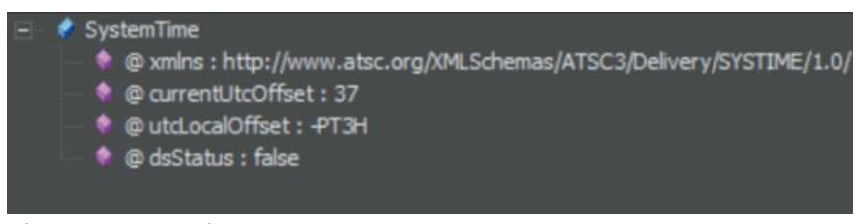

Fig. 13. SystemTime XML.

The SLS from Service 6001 (MMT) is shown in the Figure 14. Is it composed by the User Service Bundle Description (USBD) and the Complete MMT Package Table (MPT).

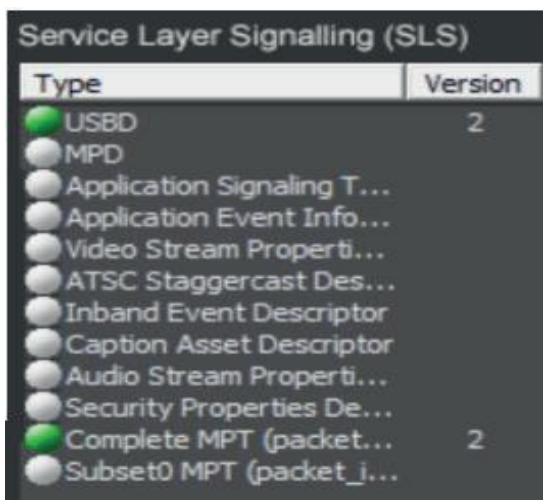

Fig. 14. SLS Signalling information - Service 6001 (MMTP).
The USBD links the MPU components to allow audio and video playback. The Figure 15 presents the USBD information.

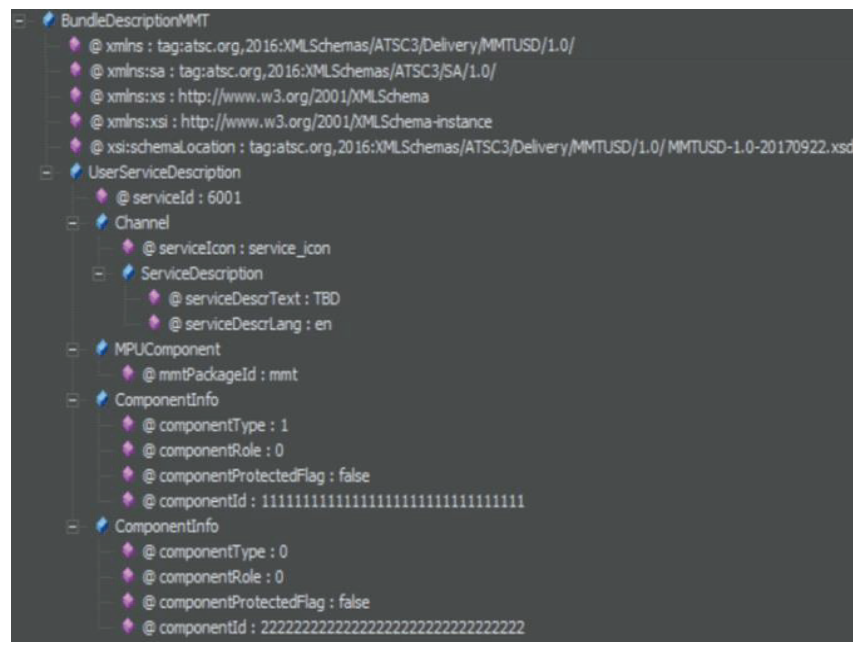

Fig. 15. USBD - Service 6001 (MMTP).

The Complete MPT contains the information in order to permit the consumption of the received packages and is presented is Figure 16.

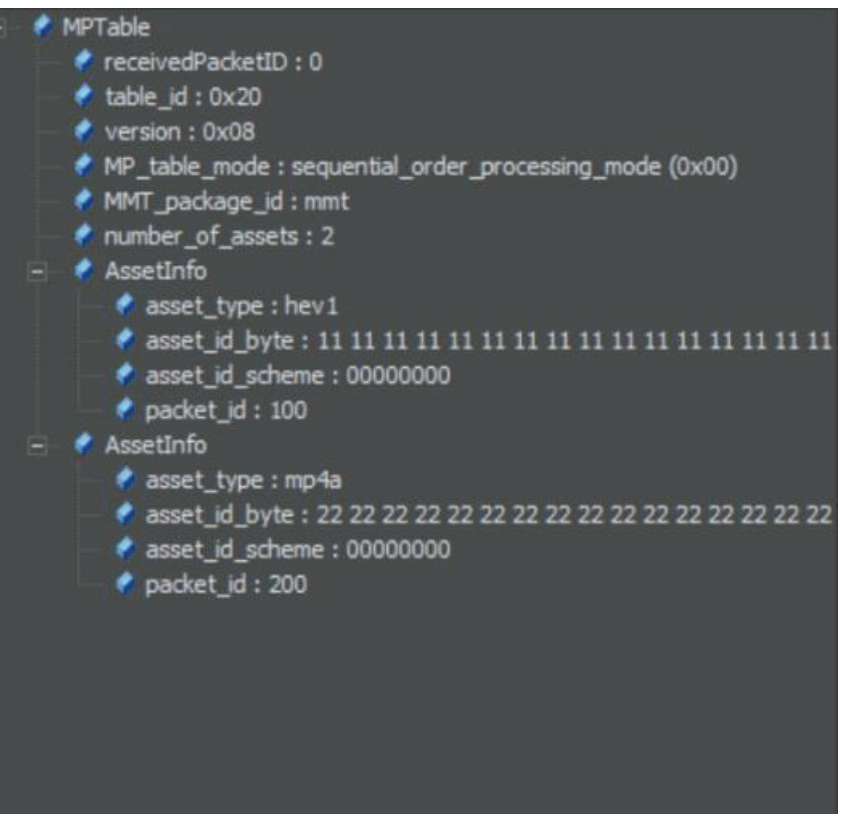

Fig. 16. Complete MPT - Service 6001 (MMTP).

The SLS from Service 6002 (ROUTE/DASH) is shown in Figure 17. This signalling is composed by the USBD, the Service-based Transport Session Instance Description (STSID) and the Media Presentation Description (MPD). 


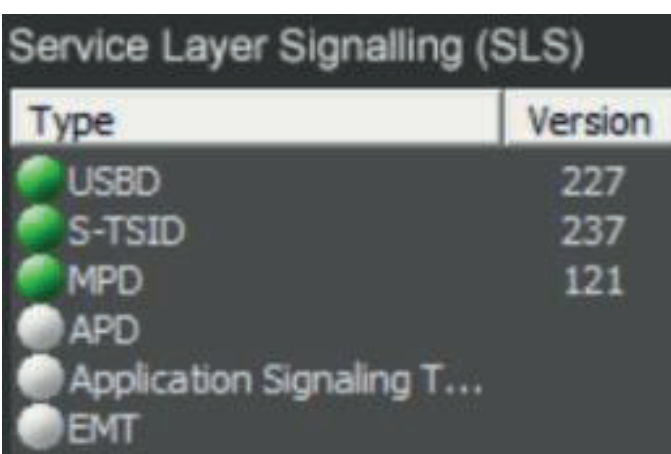

Fig. 17. SLS Signalling information - Service 6002 (ROUTE).

Figure 18 presents the USBD for service 6002 (ROUTE/DASH), in which BasePattern contains a character pattern to be used by the ATSC 3.0 receiver that matches with any portion of the Segment URL (Uniform Resource Locator) used by the DASH client.

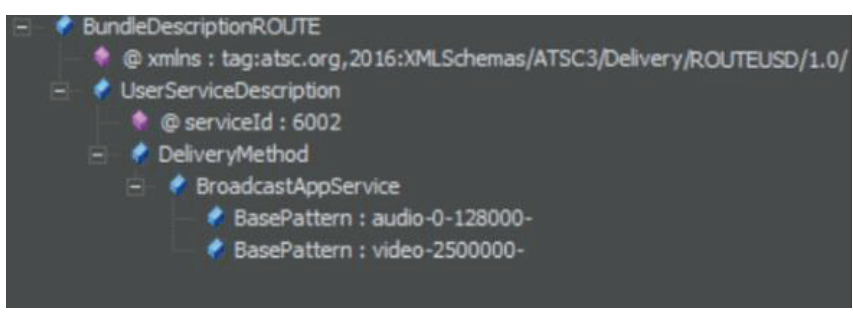

Fig. 18. USBD - Service 6002 (ROUTE).

Figure 19 shows the S-TSID information. This signalling lists the RSs and LSs, where the SrcFlow contain elements that provide information about the $\mathrm{A} / \mathrm{V}$ flow from service 6002 .

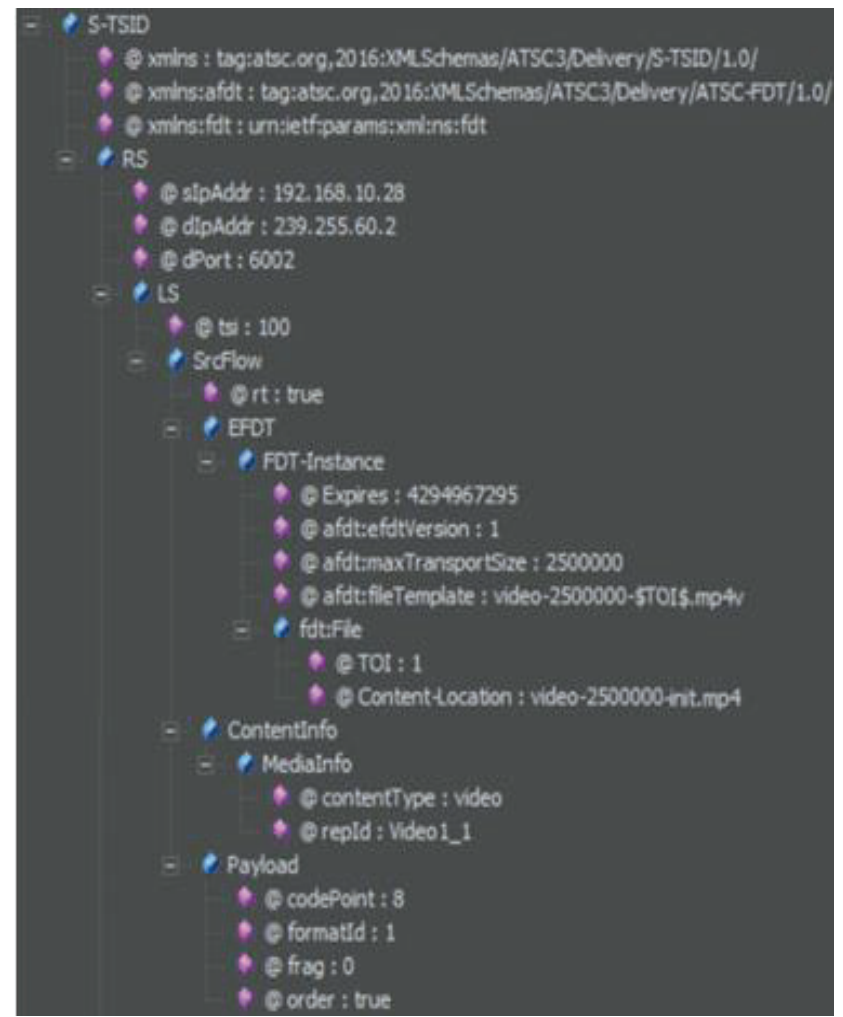

Fig. 19. S-TSID - Service 6002 (ROUTE).

Figure 20 shows the MPD table for service 6002. This signalling presents general media information.

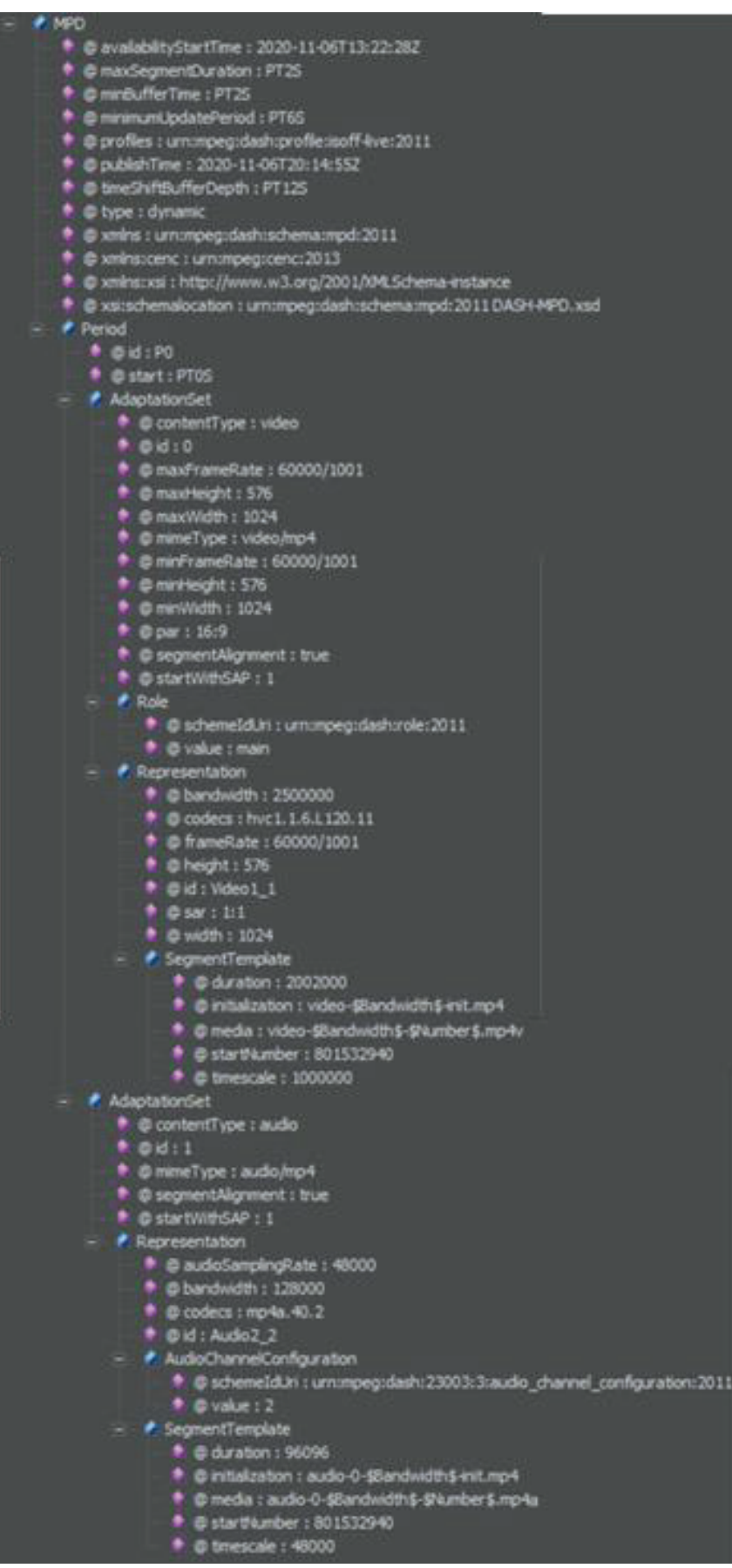

Fig. 20. MPD - Service 6002 (ROUTE).

\section{CONCLUSION}

From a conception point of view, it is possible to observe that these standards were initially developed for different purposes. MMT was developed due to new broadcast systems that needed IP connection to work with hybrid services, while DASH itself was designed for modern broadband applications. Then, ROUTE was developed to enable the delivery of DASH over broadcast, and therefore making ROUTE/DASH also compatible with hybrid systems. This resulted in distinct architectures.

Both methods are compatible with ISOBMFF and ALFEC, and the delivery protocol used in each service was signalled in slsProtocol. Also, they are both agnostic to media codecs, hence allowing future extensions.

MPEG-DASH uses MPD for content description, while MMT can also use a combination of CI and HTML5. 
For the setup configuration used for this paper, ROUTE presented a larger overhead (since the Signal Rate shown in Figure 9 was larger for ROUTE).

For this configuration, the stream from the ROUTE service was delayed in relation to the MMTP service.

MPEG-DASH has been vastly deployed, due to its popularity for broadband applications, so there are many more tools and equipment compatible with it.

For future works, some possibilities are to test with other $\mathrm{A} / \mathrm{V}$ sources, and for different configurations, such as targeted advertising, multi-view, multiple audio streams, and with additional services like the emergency alert. This would provide a more complete comparison of these methods, and possibly allow to understand if the delay observed in this paper is constant. A more complete analysis of each protocol could also contribute for a better comprehension of their characteristics. And, as a final opportunity, different setups could be elaborated to observe other aspects, such as measuring the latency of these systems for the scenarios mentioned above.

\section{ACKNOWLEDGMENT}

The authors would like to thank PPGEEC and their colleagues at Mackenzie's Digital TV Research Laboratory.

\section{REFERENCES}

[1] K. Park, Y. Lim and D. Y; Suh, "Delivery of ATSC 3.0 Services With MPEG Media Transport Standard Considering Redistribution in MPEG-2 TS Format," IEEE Transactions on Broadcasting, vol. 62, no. 1, March 2016.

[2] C. Diniz, C. Akamine and G. d. M. Valeira, "Development of MMT Analyzer for ATSC 3.0", 2019 IEEE International

Symposium on Broadband Multimedia Systems and Broadcasting (BMSB), pp. 1-5, 2019.

[3] Y. Lim, "MMT, new alternative to MPEG-2 TS and RTP," 2013 IEEE International Symposium on Broadband Multimedia Systems and Broadcasting (BMSB), pp. 1-5, 2013.

[4] Y. Lim, S. Aoki, I. Bouazizi and J. Song, "New MPEG Transport Standard for Next Generation Hybrid Broadcasting," IEEE Transactions on Broadcasting, vol. 60, no. 2, June 2014.

[5] ATSC, "ATSC Standard: Signaling, Delivery, Synchronization, and Error Protection with Amendment No. 1", Doc. A/331:2020, 27 October 2020.

[6] ITU-R, "Satellite transmissions for UHDTV satellite broadcasting”, Report ITU-R BO.2397-0, October 2016.

[7] I. Sodagar, "The MPEG-DASH Standard", IEEE Multimedia, vol. 18, no. 4, 2011, pp. 62-67.

[8] Bitmovin, "Why YouTube \& Netflix use MPEG-DASH in HTML5" [Online]. Available at $<$ https://bitmovin.com/statusmpeg-dash-today-youtube-netflix-use-html5-beyond/>. Accessed on November 2020

[9] G. K. Walker, T. Stockhammer, G. Mandyam, Y. K. Wang and C. Lo, "ROUTE/DASH IP Streaming-Based System for Delivery of Broadcast, Broadband and Hybrid Services", IEEE Transactions on Broadcasting, vol. 62, no. 1, pp. 328-337, March 2016

[10] ATSC, “ATSC Standard: A/300:2020, ATSC 3.0 System", 15 May 2020

[11] L. Fay, L. Michael, D. Gomez-Barquero, N. Ammar and M. W. Caldwell, "An Overview of the ATSC 3.0 Physical Layer Specification", IEEE Transactions on Broadcasting, vol. 62, no. 1, March 2016

[12] R. Chernock, D. Gomez-Barquero, J. Whitaker, S. Park and Y. Wu, "ATSC 3.0 Next Generation Digital TV Standard - An Overview and Preview of the Issue", IEEE Transactions on Broadcasting, vol. 62, no.1, March 2016

[13] Information technology - High efficiency coding and media delivery in heterogeneous environments - Part 1: MPEG media transport (MMT), ISO/IEC 23008-1:2017, August 2017.

[14] IETF, "RFC 5651: Layered Coding Transport (LCT) Building Block", October 2009. [Online]. Available at

$<$ https://tools.ietf.org $/$ html//rfc5651>. Accessed on November 2020
[15] ATEME, “TITAN Live User Manual”, Release 4.1.22, June 2020.

[16] IETF, "RFC 4918: HTTP Extensions for Web Distributed Authoring and Versioning (WebDAV)", June 2007. [Online]. Available at $<\mathrm{https}: / /$ tools.ietf.org $/ \mathrm{html} / \mathrm{rfc} 4918>$. Accessed on November 2020.

[17] ATSC, "ATSC Recommended Practice: Techniques for Signaling, Delivery, and Synchronization, with Amendment No. 1", Doc. A/351:2019 20 August 2019, Amendment No. 1 approved 20 January 2020.

[18] ATSC, "ATSC Standard: Scheduler/Studio to Transmitter Link", Doc. A/324:2018 5 January 2018.

[19] MP4RA, Official Registration Authority for the ISOBMFF family of standards. Available at $<$ http://mp4ra.org/>. Accessed on November 2020.

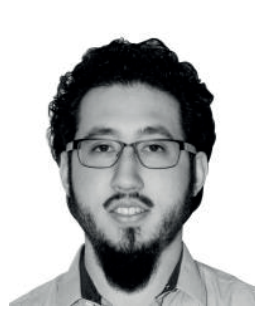

Allan Seiti Sassaqui Chaubet was born in São Paulo, in 1994. He received the B.Sc. degree in electrical engineering in 2019 from Mackenzie Presbyterian University, São Paulo, Brazil, with a sandwich period at Rose-Hulman Institute of Technology, Terre Haute, IN. $\mathrm{He}$ is currently pursuing the Ph.D. degree in electrical engineering at Mackenzie Presbyterian University.

He was an intern at the Digital TV Research Laboratory of Mackenzie Presbyterian University, where he later participated in two development projects. His research interests include broadcast television with emphasis on the transport layer.

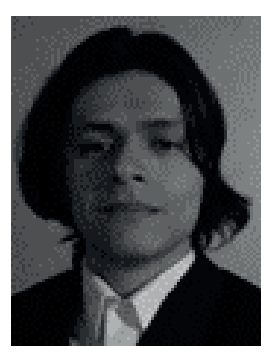

George Henrique Maranhão Garcia de Oliveira received the B.Sc. and M.Sc. degrees in electrical engineering from Mackenzie Presbyterian University, São Paulo, Brazil, in 2014 and 2016, respectively. He is currently pursuing the Ph.D. degree in electrical engineering at Mackenzie Presbyterian University.

He has experience in electrical engineering with emphasis on characterization of ISDB-T receivers, testing and configuration of SFN networks and LTE interference tests on digital TV. He also has experience in Electrical, Magnetic and Electronic Measurements. His fields of study are broadcasting, Error correction codes and Software Defined Radio.

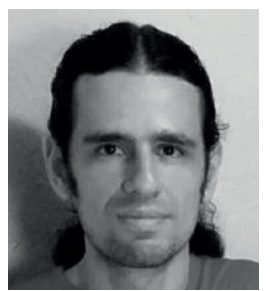

Gustavo de Melo Valeira received his B.Sc., M.Sc. and Ph.D. degrees in Electrical Engineering from Mackenzie Presbyterian University, São Paulo, Brazil, in 2007, 2010 and 2015, respectively.

He has been a researcher in the Digital TV Research Laboratory at Mackenzie Presbyterian University since 2008 and his primary research area is on FPGA and embedded processors programming for digital television. He also had the opportunity to test, work and study the ISDB-T system, including modulation, multiplexing/re-multiplexing and BTS compressor/decompressor parts, since the first transmission of ISDB-T in Brazil. His research interests include broadcast television and FPGA programming. 


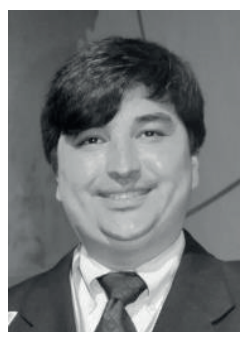

Cristiano Akamine received a Ph.D. degree in electrical engineering from the State University of Campinas, Brazil, in 2011.

$\mathrm{He}$ is a Professor at Mackenzie Presbyterian University, where he is a Coordinator of the Digital TV Research Laboratory. He is a member of the Board of the Brazilian Digital Terrestrial Television Forum and Society of Brazilian Broadcast Engineers (SET). He works in the ISDB-TB broadcasting standardization and holds several patents, licensing of intellectual property, numerous articles published. He has also served as a reviewer for several periodicals and conferences. He has participated as a Guest Editor in the Special Issue Point-to-Multipoint Communications and Broadcasting in 5G of IEEE Communications Magazine and the Special Issue on 5G for Broadband Multimedia Systems and Broadcasting of IEEE Transactions on Broadcasting. His research currently focuses on digital terrestrial broadcasting, software-defined radio, channel codes, embedded systems, and $5 \mathrm{G}$. 